\title{
MicroRNA-766 targeting regulation of SOX6 expression promoted cell proliferation of human colorectal cancer
}

This article was published in the following Dove Press journal:

OncoTargets and Therapy

20 October 2015

Number of times this article has been viewed

\section{Yong-chao $\mathrm{Li}^{\prime}$ \\ Chang-feng $\mathrm{Li}^{2}$ \\ Li-bo Chen ${ }^{2}$ \\ Dan-dan $\mathrm{Li}^{2}$ \\ Lei Yang ${ }^{2}$ \\ Jing-peng Jin ${ }^{2}$ \\ Bin Zhang ${ }^{2}$}

'Department of Gastrointestinal Surgery, ${ }^{2}$ Department of Endoscopy Center, China-Japan Union Hospital of Jilin University, Changchun, People's Republic of China
Correspondence: Chang-feng Li Department of Endoscopy Center, China-Japan Union Hospital of Jilin University, 126 Xiantai St, Erdao, Changchun, Jilin I30033, People's Republic of China

$\mathrm{Tel}+8643184641026$

Email changfenglijilin@163.com

\begin{abstract}
MicroRNAs (miRNAs) have emerged as important regulators of cancer-cell biological processes. Previous studies have shown that miR-766 plays an important role in a variety of biological processes in various human cancers. However, the underlying mechanism of miR-766 in colorectal cancer (CRC) cells remains unclear. In this study, we investigated miR-766's role in CRC cell proliferation. Polymerase chain reaction results showed that miR766 expression was significantly upregulated in CRC tissues and cells. Ectopic expression of miR-766 promoted cell growth and anchorage-independent growth in CRC cells. Bioinformatic analysis predicted SOX6, a potential target of miR-766, acting as a tumor suppressor. Luciferase reporter assay results demonstrated that miR-766 directly bound to the $3^{\prime}$-untranslated region of SOX6. Overexpression of miR-766 suppressed SOX6 expression, resulting in the downregulation of $\mathrm{p} 21$ and upregulation of cyclin D1. In a further experiment, SOX6-silenced SW480 cells transfected with miR-766 promoted cell growth, suggesting that downregulation of SOX6 was required for miR-766-induced CRC cell proliferation. Taken together, these results suggested that miR-766 represents an onco-miRNA and participates in the development of CRC by modulating SOX6 expression.
\end{abstract}

Keywords: miR-766, colorectal cancer, SOX6, cell proliferation

\section{Introduction}

Colorectal cancer (CRC) is the third-leading cause of cancer-related deaths worldwide. ${ }^{1}$ However, the molecular underpinnings of CRC proliferation remain incompletely understood. Recently, microRNAs (miRNAs) were discovered to be endogenous noncoding small RNAs ( $\sim 22$ nucleotides) that play an important role in regulating the diverse biological progression of multiple cancers, such as proliferation, apoptosis, differentiation, and metabolism..$^{2-4}$ In view of the close relationship between the biological processes of cancers and miRNAs, miRNAs are presently considered new therapeutic targets for the treatment of cancer. ${ }^{5-8}$

Growing evidence indicates that miR-766 acts as a tumor promoter or suppressor in multiple cancers. ${ }^{9-11}$ However, the relationship between CRC and miR-766 remains unclear. In this study, we investigated the role of miR-766 in CRC. Our results demonstrated that overexpression of miR-766 effectively promoted cell proliferation by repressing SOX6 in CRC. Further experimentation demonstrated that SOX6 was a bona fide target of miR-766. Taken together, our data demonstrated that miR-766 indeed acted as a proliferation promoter through the downregulation of SOX6. 


\section{Materials and methods}

\section{Clinical specimens}

Eight paired human CRC tissue samples and matched tumor-adjacent tissue were obtained from CRC patients and histopathologically diagnosed at the China-Japan Union Hospital of Jilin University (Changchun, People's Republic of China). The study was approved by the ethics committee of the ChinaJapan Union Hospital of Jilin University. Written informed consent was obtained from all patients. Tissue samples were collected at surgery, immediately frozen in liquid nitrogen, and stored until total RNAs or proteins were extracted.

\section{Cell culture}

Human CRC cell lines HT-29, COLO205, SW620, SW403, KM202L, SW480, and COLO320DM and normal colonic cell line FHC were purchased from National Rodent Laboratory Animal Resource (Shanghai, People's Republic of China). All CRC cell lines were grown in Dulbecco's Modified Eagle's Medium (Thermo Fisher Scientific, Waltham, MA, USA) supplemented with $10 \%$ fetal bovine serum (Sigma-Aldrich Co, St Louis, MO, USA), 100 U/mL of penicillin-streptomycin (Thermo Fisher Scientific), and normal colon FHC cells were grown in Dulbecco's Modified Eagle's Medium/F-12 medium with $10 \%$ fetal bovine serum, $10 \mathrm{ng} / \mathrm{mL}$ cholera toxin, $5 \mu \mathrm{g} / \mathrm{mL}$ transferrin, $5 \mu \mathrm{g} / \mathrm{mL}$ insulin, $100 \mathrm{ng} / \mathrm{mL}$ hydrocortisone, and an extra $10 \mathrm{mM}$ HEPES. Cell lines were cultured in a humidified incubator at $37^{\circ} \mathrm{C}$ in an atmosphere of $5 \% \mathrm{CO}_{2}$ and $95 \%$ air. The protocols in this study in regards to human cell lines were approved by China-Japan Union Hospital of Jilin University (Changchun, China).

\section{Plasmids, small interfering RNA, and transfection}

MiR-766 mimic, miR-766 inhibitor, and negative control were purchased from GeneCopoeia (Rockville, MD, USA). For depletion of SOX6, SOX6 small interfering RNA (siRNA) and negative control were purchased from Qiagen NV (Venlo, the Netherlands). Transfection of plasmids and siRNAs was performed using Lipofectamine 2000 (Thermo Fisher Scientific), according to the manufacturer's protocol.

\section{RNA extraction and quantitative real- time PCR}

Total RNA was extracted from culture cells and patient samples using Trizol (Thermo Fisher Scientific) as recommended by the manufacturer's instructions, and quantitative real-time polymerase chain reaction (qRT-PCR) for miRNA was performed using the TaqMan miRNA reverse-transcription kit (Thermo Fisher Scientific). The relative miR-766 expression levels after normalization to U6 small nuclear RNA were calculated using 2-[(CtofmiR-766)-(CtofU6)]. The qRT-PCR assays were performed using an SYBR kit (Qiagen) using a LightCycler system. The primers selected were as follows: cyclin D1 forward 5'-TCCTCTCCAAAATGC CAGAG-3', cyclin D1 reverse 5'-GGCGGATTGGAAATGAACTT-3'; p21 forward 5'-CATGGGTTCTGACGGACAT-3', p21 reverse 5'-AGTCAGTTCCTTGTGGAGCC-3'. Expression data were normalized to the geometric mean of GAPDH (forward primer 5'-GACGGCCGCATCTTCTTGT-3', reverse primer 3'-CACACCGACCTTCACCATTTT-5') to control the variability in expression levels and calculated as $2^{-[(\text {CtofCyclinD1andp21)-(CtofGAPDH)] }}$.

\section{Western blotting}

Protein lysates were lysed in radioimmunoprecipitation assay buffer (Beyotime, Shanghai, People's Republic of China); equal quantities $(50 \mu \mathrm{g})$ of protein were subjected to $10 \%$ sodium dodecyl sulfate polyacrylamide gel electrophoresis, and then gels were transferred onto polyvinylidene difluoride membranes for 2 hours at $4^{\circ} \mathrm{C}$, run at a current of $125 \mathrm{~mA}$, and blocked for 2 hours. The membrane was incubated overnight with anti-SOX6 (1:500; Abcam, Cambridge, MA, USA), anti-cyclin D1, anti-p21 (1:1,000; Cell Signaling Technology, Danvers, MA, USA), and $\alpha$-tubulin (1:2,000 dilution; Abcam) was used as a reference protein. Membranes were further incubated with corresponding horseradish peroxidase-conjugated secondary antibodies for 2 hours at room temperature, and blots were visualized using an ECL Kit (Beyotime).

\section{MTT assay and colony-formation assay}

Cell growth was measured by MTT assay (Sigma-Aldrich) according to the manufacturer's instructions. Briefly, $5 \times 10^{3}$ transfected cells were seeded in 96-well plates and cultured for $1,2,3$, and 4 days. Exactly $20 \mu \mathrm{L}$ of $5 \mathrm{mg} / \mathrm{mL}$ MTT solution (Sigma-Aldrich) was added to the test well and incubated for 4 hours, and then the culture medium was removed and $150 \mu \mathrm{L}$ dimethyl sulfoxide (Sigma-Aldrich) was added. Optical density at $490 \mathrm{~nm}$ was measured using a microplate reader (Molecular Devices LLC, Sunnyvale, CA, USA).

For the colony-formation assay, SW480 cells after transfection were plated in six-well plates ( 1,000 cells per well) and incubated for 10 days. Cells were fixed with methanol and stained with $0.1 \%$ crystal violet. Visible colonies were manually counted.

\section{Anchorage-independent growth assay}

Two milliliters complete medium supplemented with $0.5 \%$ agar (Sigma-Aldrich) was added to each well of the six-well plates to form a layer of base gel. Plates were incubated at 
$37^{\circ} \mathrm{C}$ in $5 \% \mathrm{CO}_{2}$ for 14 days. Colonies were observed and counted under microscope.

\section{Luciferase assays}

The PPP2R2A open reading frames with 3 '-untranslated region (UTR) was cloned into pGL3. Vectors were purchased from GeneCopoeia. The primers selected were as follows: SOX6-3'UTR-wt-up: 5'-GCCCCGCGGTGGCTCC ACAATTACATCAGC-3'; SOX6-3'UTR-wt-down: $3^{\prime}$-GCCCTGCAGCATAAAATCACTATGTACA CAGGA- $5^{\prime}$.

SW480 cells $\left(5 \times 10^{4} /\right.$ well $)$ were cultured in 24 -well plates and cotransfected with pGL3-PPP2R2A-3'-UTR and $100 \mathrm{nM}$ of miR-766 or miR-766-in or miR-766-mutant (mut) or control mimics using Lipofectamine 2000 reagent. Luciferase and Renilla activities were assayed 48 hours after transfection using the dual-luciferase assay kit to the manufacturer's protocol.

\section{Statistical analysis}

All statistical analyses except for microarray data were performed using SPSS 17.0 (SPSS Inc, Chicago, IL, USA). Student's $t$-test was used to evaluate the significance of the differences between two groups of data in all the pertinent experiments. $P<0.05$ (using a two-tailed paired $t$-test) was thought to be significantly different for two groups of data.

\section{Results}

\section{MiR-766 expression elevated in CRC tissues and CRC cell lines}

To investigate the potential roles of miR-766 in CRC development, results of RT-PCR analysis revealed that compared with the matched tumor-adjacent tissue, miR-766 expression was differentially upregulated in the CRC tissues, and all eight tested CRC cell lines showed significantly upregulated expression of miR-766 compared to the normal colonic cell line FHC (Figure 1).

\section{MiR-766 promoted CRC cell proliferation}

To investigate the role of miR-766 in CRC cell proliferation further, SW480 cells were transfected with miR-766 mimics, miR-766 inhibitor, or the relative controls. Relative miR-766 expression was verified using RT-PCR (Figures 2A and 3A). Ectopic expression of miR-766 significantly increased the growth rate of SW480 cells from day 2 of the experiment (Figure 2B). Colony-formation assay showed that upregulation of miR-766 promoted the colony-formation capacity of SW480 cells (Figure 2C). Strikingly, we found that overexpression of miR-766 in SW480 cells significantly enhanced their anchorage-independent growth ability (Figure 2D). In contrast, miR-766-in showed the opposite effect (Figure 3B-D). Collectively, these results showed that miR-766 enhanced the proliferation of SW480 cells in vitro.

\section{MiR-766 directly targeted SOX6 by binding to its $3^{\prime}$-UTR}

As SOX6 was a binding target of miR-766 predicted by TargetScan (Figure 4A), SW480 cells were transfected with miR-766 mimic, miR-766-in, or the respective controls. The results of Western blots revealed that the expression of SOX6 on protein level was remarkably decreased after the overexpression of miR-766 (Figure 4B), while miR-766-in
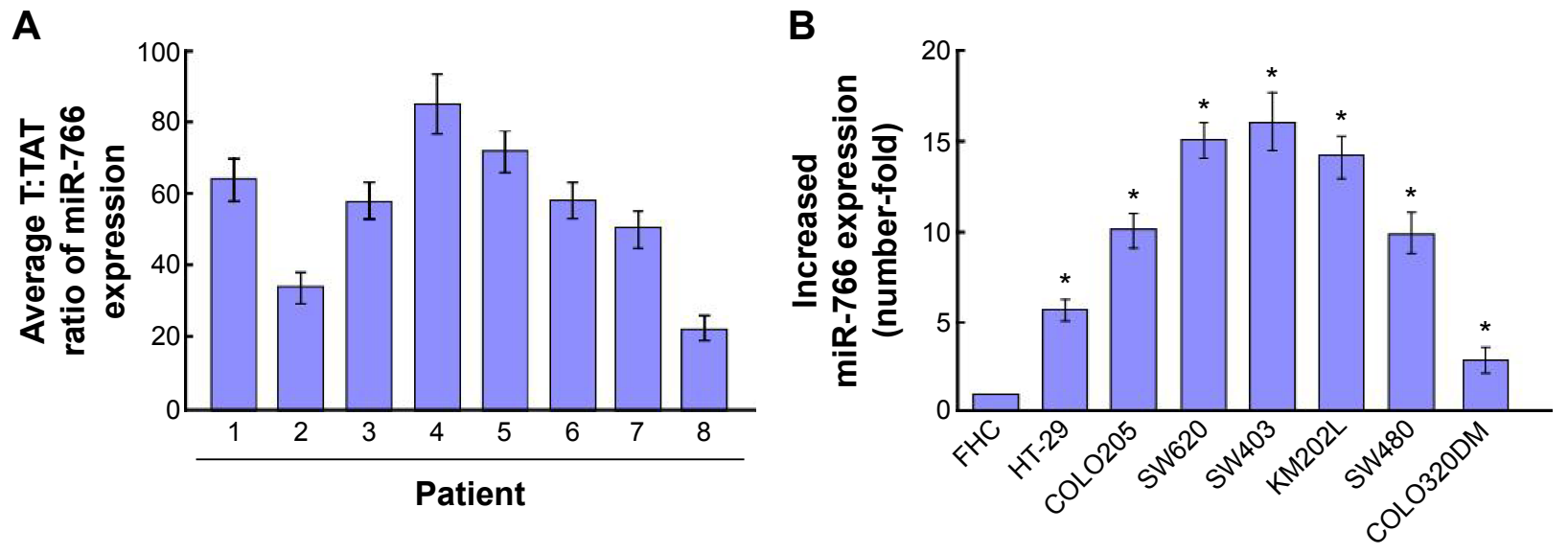

Figure I Expression of miR-766 in human colorectal cancer (CRC) tissues and cell lines.

Notes: (A) Relative miR-766 messenger RNA-expression levels in eight paired primary CRC tissue (T) samples and matched tumor-adjacent tissue (TAT) from the same patient were detected by polymerase chain reaction (PCR) analysis. (B) Real-time PCR analysis of miR-766 expression in FHC cells and CRC cell lines, including HT-29, COLO205, SW620, SW403, KM202L, SW480, and COLO320DM. Experiments were repeated at least three times. Each bar represents the mean of three independent experiments. $* P<0.05$. 
A

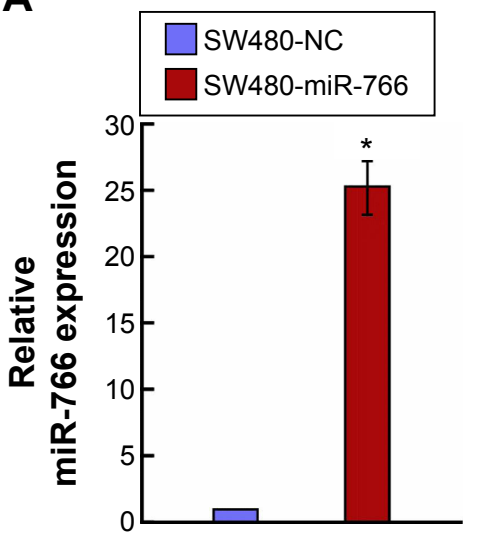

C
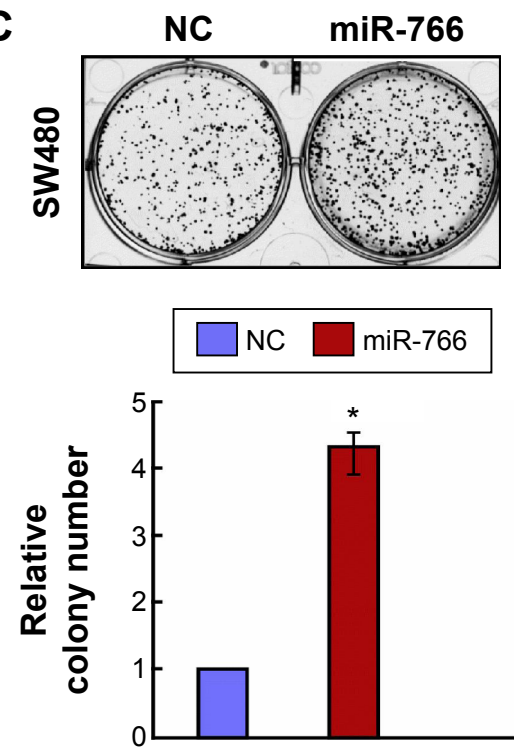

B

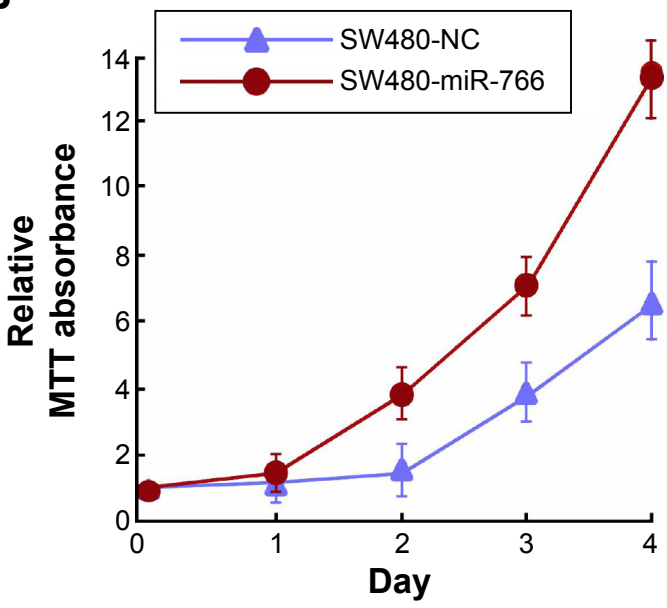

D
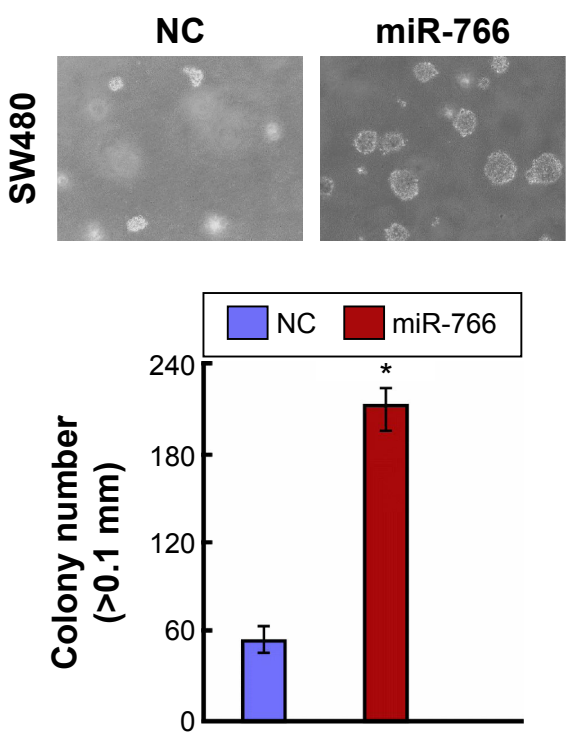

Figure 2 miR-766 upregulation promoted CRC cell proliferation.

Notes: (A) Validation of miR-766 expression levels after transfection by PCR analysis. (B) MTT assays revealed that upregulation of miR-766 promoted growth of SW480 cells. (C) Representative micrographs (left) and quantification (right) of crystal violet-stained cell colonies. (D) Upregulation of miR-766 promoted the anchorage-independent growth of SW480 cells. Representative micrographs (left) and quantification of colonies that were $>0.1$ mm (right). Each bar represents the mean of three independent experiments. $* P<0.05$.

Abbreviations: CRC, colorectal cancer; PCR, polymerase chain reaction; NC, negative control.

significantly increased SOX6 protein expression. For the purpose of identifying SOX6 as a direct target of miR-766, SW480 cells were transfected with SOX6 3'-UTR (wild or mutant) vector and miR-766 mimic, miR-766-in, or miRNA negative controls. Dual-luciferase reporter assay results showed that reduction in luciferase activity was observed in miR-766 -transfected SW480 cells, whereas the repressive effect of miR-766-in increased wild-type SOX6 luciferase activity. Meanwhile, the luciferase activities of cells cotransfected with miR-766 and SOX6 3'-UTR-mut vector were unaffected. Our data indicated that SOX6 might be involved in cellular proliferation of CRC. We detected that the SOX6 downstream genes, messenger RNA, and protein expression of $\mathrm{p} 21$ were remarkably downregulated and cyclin D1 expression was remarkably upregulated by miR-766 (Figure 4D). In contrast, the expression level of p21 was upregulated and cyclin D1 downregulated in SW480 cells transfected with miR-766-in (Figure 4E). Collectively, these results revealed that SOX6 was a bona fide target of miR-766.

\section{SOX6 suppression required for miR-766- induced cell proliferation in CRC}

To investigate the role of SOX6 reduction in CRC proliferation further, we first examined the effects of SOX6 
A

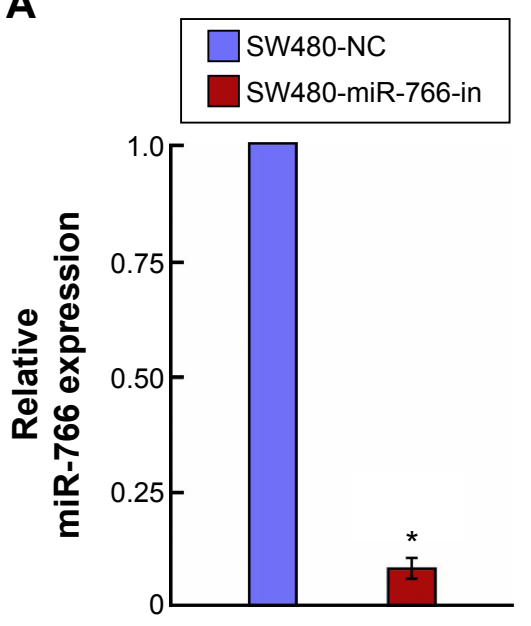

C
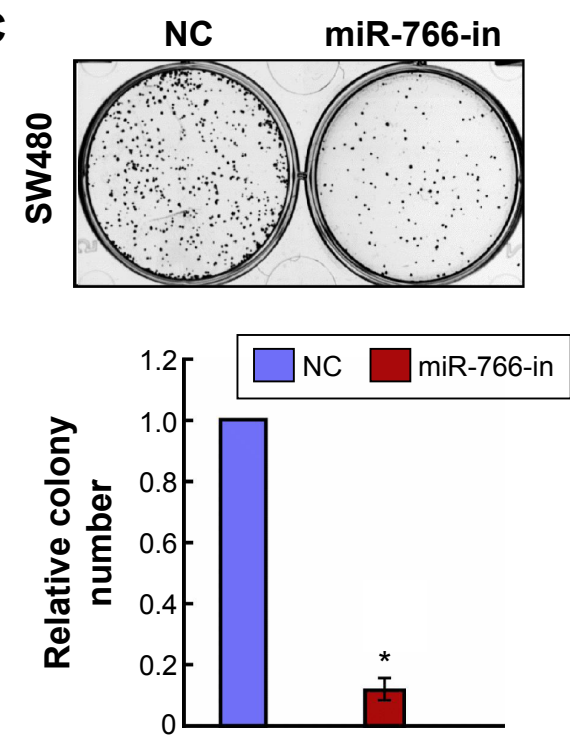

B
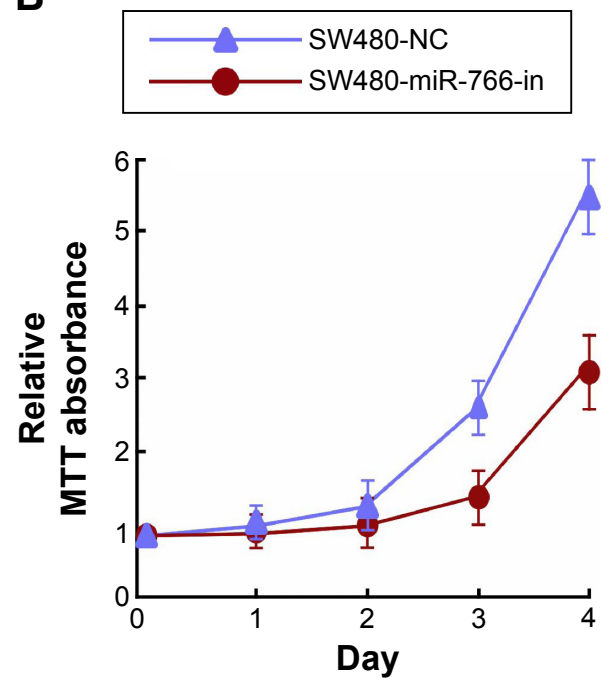

D
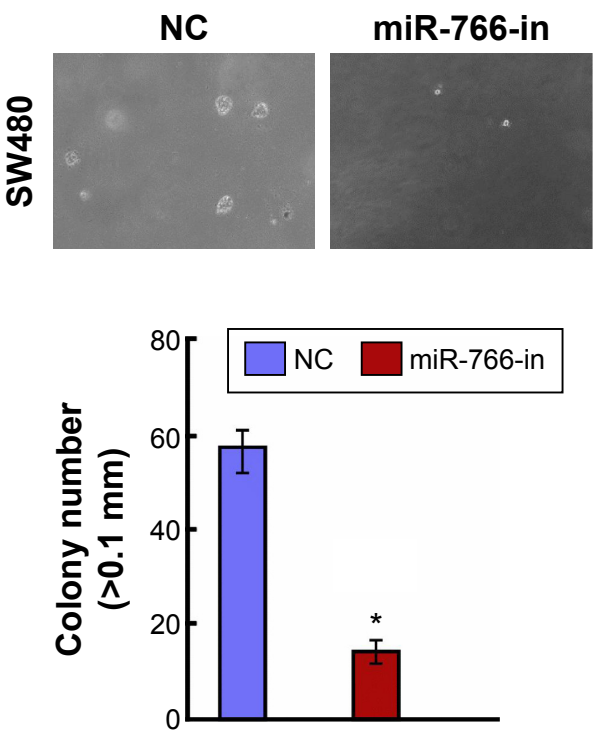

Figure 3 Inhibition of miR-766 inhibited CRC cell proliferation.

Notes: (A) Validation of miR-766 expression levels after transfection by PCR analysis. (B) MTT assays revealed that inhibition of miR-766 suppressed the growth of SW480 cells. (C) Representative micrographs (left) and quantification (right) of crystal violet-stained cell colonies. (D) Inhibition of miR-766 inhibited the anchorage-independent growth of SW480 cells. Representative micrographs (left) and quantification of colonies that were $>0.1 \mathrm{~mm}$ (right). Each bar represents the mean of three independent experiments. $* P<0.05$.

Abbreviations: CRC, colorectal cancer; PCR, polymerase chain reaction; NC, negative control.

downregulation on CRC cell proliferation. Results of Western blot analysis indicated that silencing SOX6 effectively decreased SOX6 protein expression in SW480 cells transfected with miR-766-in (Figure 5A). Results of colony formation and anchorage-independent growth assays demonstrated that knockdown of SOX6 effectively counteracted the proliferation arrest by miR-766 (Figure 5B and C). Taken together, these results indicated that SOX6 was an important target of miR-766 and was involved in miR-766-regulated CRC cell proliferation.

\section{Discussion}

In the present study, we noted that miR-766 expression was significantly upregulated in CRC tissues and cells. Moreover, overexpression of miR-766 enhanced the cell growth of CRC cells, whereas miR-766-in showed the opposite effect, probably through repressing SOX6 expression. The downregulation of SOX6 by miR-766 led to upregulation of $\mathrm{p} 21$ and downregulation of cyclin D1. Our results showed that miR-766 might play an essential role via the SOX6-mediated pathway in the progress of CRC. 
A

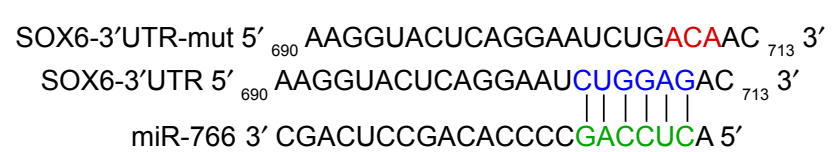

C

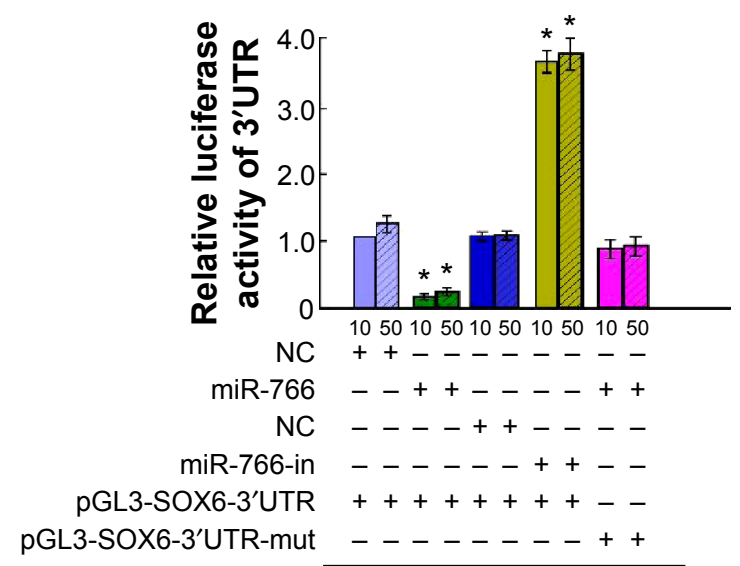

B

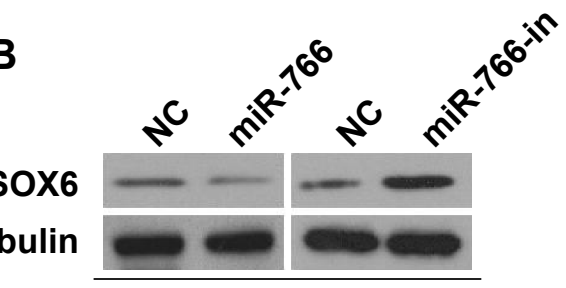

SW480

D

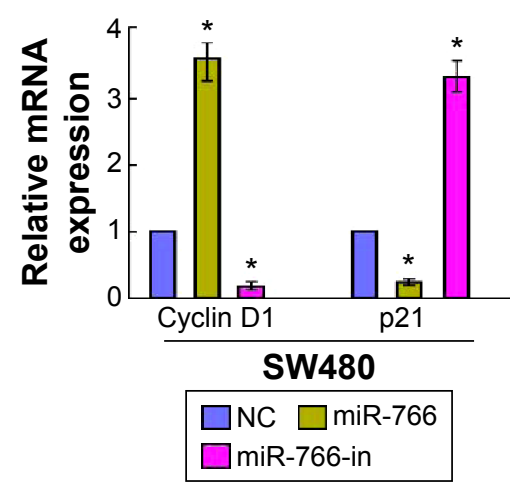

SW480

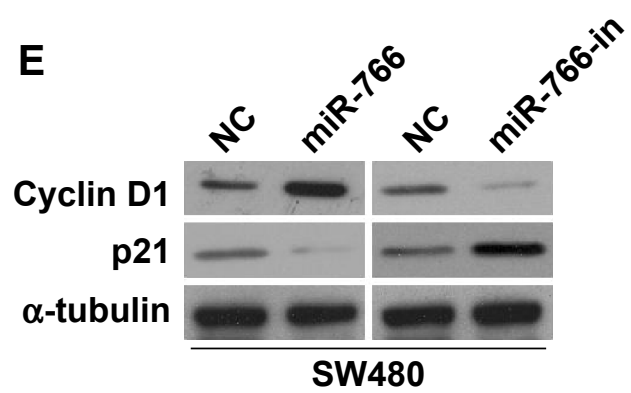

Figure 4 miR-766 suppressed SOX6 expression by directly targeting the SOX6 3'-UTR and altered levels of proteins related to proliferation in SW480 cells.

Notes: (A) Predicted miR-766 target sequence in the 3'-UTR of SOX6 (SOX6-3'-UTR) and positions of three mutated nucleotides (red) in SOX6-3'-UTR-mutant (mut). (B) SOX6 protein expression in SW480 cells transfected with miR-766 or the miR-766 inhibitor were detected by Western blotting analysis. $\alpha$-tubulin served as the loading control. (C) Luciferase reporter assay of SW480 cells transfected with the PGL3-SOX6-3'-UTR reporter or pGL3-SOX6-3'-UTR-mut reporter and miR-766 or miR-766-in with increasing amounts (10 and $50 \mathrm{nM}$ ) of oligonucleotides. (D) Real-time PCR analysis of expression of cyclin DI and p2I in SW480 cells. (E) Western blotting analysis of protein expression of cyclin DI and $\mathrm{P} 2 \mathrm{I}$ in SW480 cells. $\alpha$-tubulin served as the loading control. $* \mathrm{P}<0.05$.

Abbreviations: UTR, untranslated region; PCR, polymerase chain reaction; NC, negative control; mRNA, messenger RNA.

Growing evidence suggests that miRNAs play essential roles in a broad range of biological processes, including cell proliferation, differentiation, angiogenesis, migration, and invasion. ${ }^{12-15}$ Recently, aberrant expressions of miRNAs have been increasingly investigated. However, it was uncertain whether miR-766 was associated with the development of CRC. In this study, we found that miR-766 negatively regulated SOX6 expression by targeting the SOX6 3'-UTR. SOX6, a member of the Sox transcription-factor family, plays critical roles in cell differentiation and proliferation. ${ }^{16,17}$ SOX6 is reported to have a tumor-suppressive function in cancers. ${ }^{18,19}$ Qin et al indicated that SOX6 inhibited cell proliferation by upregulating expressions of $\mathrm{p} 53$ and $\mathrm{p} 21$ and downregulating expressions of cyclin D1/CDK4, cyclin A, and $\beta$-catenin in esophageal cancer. ${ }^{20} \mathrm{Cyclin} \mathrm{D} 1$ and $\mathrm{p} 21$ are crucial in the regulation of cell proliferation, differentiation, apoptosis, and other cellular functions. ${ }^{21-23}$ In line with these reports, in our study, ectopic expression of miR-766 led to downregulation of SOX6, which resulted in the downregulation of $\mathrm{p} 21$, upregulation of cyclin D1, and thus the promotion of CRC cell proliferation.

In sum, our data demonstrated that miR-766 is a protooncogenic miRNA in CRC cells and that SOX6 is a novel and critical miRNA-766 target. Therefore, all the results indicated that miR-766 might serve as a potential therapeutic target for treating CRC. 
A

SW480-miR-766-in

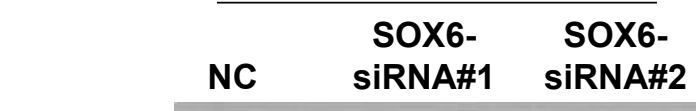

\section{sox6}

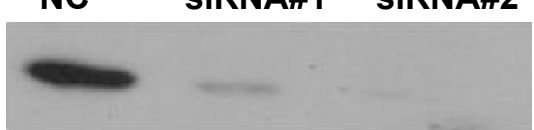

o-tubulin

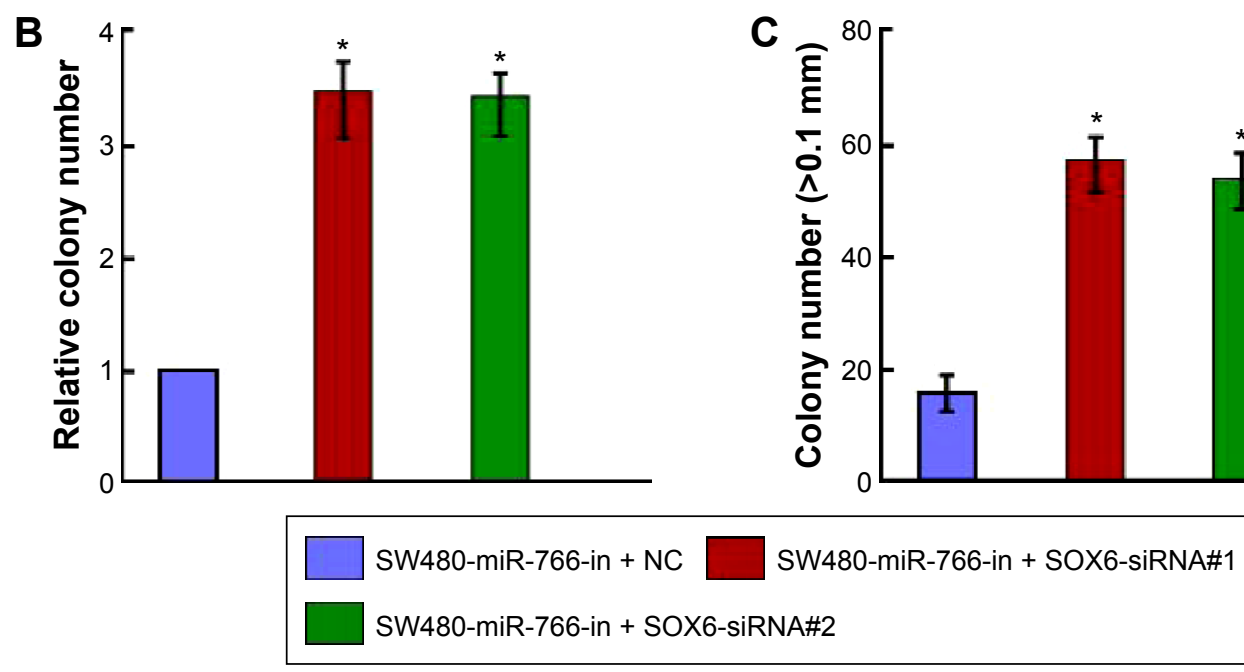

Figure 5 SOX6 downregulation was required for miR-766-induced proliferation of CRC cells.

Notes: (A) Western blot analysis verified that silencing SOX6 effectively decreased the expression of SOX6 in miR-766-in-transfected SW480 cells. (B) miR-766-intransfected SW480 cells after transfection with SOX6 siRNAs promoted cell-colony formation. (C) miR-766-in-transfected SW480 cells after transfection with SOX6 siRNAs promoted anchorage-independent growth. Representative quantification of colonies that were $>0.1 \mathrm{~mm}$. Each bar represents the mean of three independent experiments. $* P<0.05$.

Abbreviations: CRC, colorectal cancer; siRNAs, small interfering RNAs; NC, negative control.

\section{Acknowledgments}

This work was supported by the Department of Gastrointestinal Surgery and Department of Endoscopy Center, ChinaJapan Union Hospital of Jilin University, as well as funded by the Science and Technology Agency of Jilin Province (20140414002G H). All authors designed the study together, performed the experiment together, analyzed the data, and wrote the paper. All authors approved the final manuscript and agree to be accountable for all aspects of the work.

\section{Disclosure}

The authors report no conflicts of interest in this work.

\section{References}

1. Jemal A, Siegel R, Xu J, Ward E. Cancer statistics, 2010. CA Cancer J Clin. 2010;60(5):277-300.

2. Calin GA, Croce CM. MicroRNA signatures in human cancers. Nat Rev Cancer. 2006;6(11):857-866.

3. Esquela-Kerscher A, Slack FJ. Oncomirs - microRNAs with a role in cancer. Nat Rev Cancer. 2006;6(4):259-269.
4. Shukla GC, Singh J, Barik S. MicroRNAs: processing, maturation, target recognition and regulatory functions. Mol Cell Pharmacol. 2011; 3(3):83-92.

5. Cho WC. OncomiRs: the discovery and progress of microRNAs in cancers. Mol Cancer. 2007;6:60.

6. Xu L, Dai WQ, Xu XF, Wang F, He L, Guo CY. Effects of multiple-target anti-microRNA antisense oligodeoxyribonucleotides on proliferation and migration of gastric cancer cells. Asian Pac J Cancer Prev. 2012; 13(7):3203-3207.

7. Jardin F, Figeac M. MicroRNAs in lymphoma, from diagnosis to targeted therapy. Curr Opin Oncol. 2013;25(5):480-486.

8. Fiorucci G, Chiantore MV, Mangino G, Percario ZA, Affabris E, Romeo G. Cancer regulator microRNA: potential relevance in diagnosis, prognosis and treatment of cancer. Curr Med Chem. 2012;19(4): 461-474.

9. Sand M, Skrygan M, Georgas D, et al. Microarray analysis of microRNA expression in cutaneous squamous cell carcinoma. J Dermatol Sci. 2012; 68(3):119-126.

10. Li X, Shi Y, Yin Z, Xue X, Zhou B. An eight-miRNA signature as a potential biomarker for predicting survival in lung adenocarcinoma. J Transl Med. 2014;12:159.

11. Liang H, Li X, Wang L, et al. MicroRNAs contribute to promyelocyte apoptosis in As2O3-treated APL cells. Cell Physiol Biochem. 2013; 32(6):1818-1829.

12. Shen F, Cai WS, Feng Z, et al. MiR-492 contributes to cell proliferation and cell cycle of human breast cancer cells by suppressing SOX7 expression. Tumour Biol. 2015;36(3):1913-1921. 
13. Zhang W, Mao YQ, Wang H, Yin WJ, Zhu SX, Wang WC. MiR-124 suppresses cell motility and adhesion by targeting talin 1 in prostate cancer cells. Cancer Cell Int. 2015;15:49.

14. Yan B, Guo Q, Fu FJ, et al. The role of miR-29b in cancer: regulation, function, and signaling. Onco Targets Ther. 2015;8:539-548.

15. Zhang Z, Sun J, Bai Z, et al. MicroRNA-153 acts as a prognostic marker in gastric cancer and its role in cell migration and invasion. Onco Targets Ther. 2015;8:357-364.

16. Li X, Wang J, Jia Z, et al. MiR-499 regulates cell proliferation and apoptosis during late-stage cardiac differentiation via Sox6 and cyclin D1. PloS One. 2013;8(9):e74504.

17. Ohta S, Misawa A, Lefebvre V, Okano H, Kawakami Y, Toda M. Sox6 up-regulation by macrophage migration inhibitory factor promotes survival and maintenance of mouse neural stem/progenitor cells. PloS One. 2013;8(9):e74315.

18. Guo X, Yang M, Gu H, Zhao J, Zou L. Decreased expression of SOX6 confers a poor prognosis in hepatocellular carcinoma. Cancer Epidemiol. 2013;37(5):732-736.
19. Li H, Zheng D, Zhang B, et al. Mir-208 promotes cell proliferation by repressing SOX6 expression in human esophageal squamous cell carcinoma. J Transl Med. 2014;12:196.

20. Qin YR, Tang H, Xie F, et al. Characterization of tumor-suppressive function of SOX6 in human esophageal squamous cell carcinoma. Clin Cancer Res. 2011;17(1):46-55.

21. Xu C, Wu C, Xia Y, et al. WT1 promotes cell proliferation in non-small cell lung cancer cell lines through up-regulating cyclin D1 and p-pRb in vitro and in vivo. PloS One. 2013;8(8):e68837.

22. Stöckl S, Göttl C, Grifka J, Grässel S. Sox 9 modulates proliferation and expression of osteogenic markers of adipose-derived stem cells (ASC). Cell Physiol Biochem. 2013;31(4-5):703-717.

23. Liu L, Zhang H, Shi L, et al. Inhibition of Rac1 activity induces G1/S phase arrest through the GSK3/cyclin D1 pathway in human cancer cells. Oncol Rep. 2014;32(4):1395-1400.
OncoTargets and Therapy

\section{Publish your work in this journal}

OncoTargets and Therapy is an international, peer-reviewed, open access journal focusing on the pathological basis of all cancers, potential targets for therapy and treatment protocols employed to improve the management of cancer patients. The journal also focuses on the impact of management programs and new therapeutic agents and protocols on

\section{Dovepress}

patient perspectives such as quality of life, adherence and satisfaction. The manuscript management system is completely online and includes a very quick and fair peer-review system, which is all easy to use. Visit http://www.dovepress.com/testimonials.php to read real quotes from published authors. 